\title{
Broad absorption line symbiotic stars: highly ionized species in the fast outflow from MWC 560
}

\author{
Adrian B. Lucy, ${ }^{1 \star} \dagger$ Christian Knigge, ${ }^{2}$ and J. L. Sokoloski ${ }^{1}$ \\ ${ }^{1}$ Columbia University, Dept. of Astronomy, 550 West 120th Street, New York, NY 10027, U.S.A. \\ ${ }^{2}$ University of Southampton, School of Physics $\&$ Astronomy, Highfield, Southampton, SO17 1BJ, U.K.
}

\begin{abstract}
In symbiotic binaries, jets and disk winds may be integral to the physics of accretion onto white dwarfs from cool giants. The persistent outflow from symbiotic star MWC 560 ( $\equiv \mathrm{V} 694 \mathrm{Mon})$ is known to manifest as low-ionization broad absorption lines (BALs), most prominently at the Balmer transitions, and as high-ionization BALs from metastable He I*. We report the detection of higher-ionization BALs from C IV, Si IV, Nv, and He II in International Ultraviolet Explorer spectra obtained on 1990 April 29-30, when an optical outburst temporarily erased the obscuring 'iron curtain' of absorption troughs from Fe II and similar ions. The CIV and Si IV BALs reached maximum radial velocities at least $1000 \mathrm{~km} \mathrm{~s}^{-1}$ faster than contemporaneous Mg II and He II BALs; the same behaviors occur in the winds of quasars and cataclysmic variables. An iron curtain lifts to unveil high-ionization BALs during the P Cygni phase observed in some novae, suggesting by analogy a temporary switch in MWC 560 from persistent outflow to discrete mass ejection. At least three more symbiotic stars exhibit broad absorption with blue edges faster than $1500 \mathrm{~km} \mathrm{~s}^{-1}$; exclusively highionization BALs have been reported in AS 304 ( $\equiv$ V4018 Sgr), while instead transient Balmer BALs have been reported in Z And and CH Cyg. These BAL-producing fast outflows can have wider opening angles than has been previously supposed. BAL symbiotics are short-timescale laboratories for their giga-scale analogs, broad absorption line quasars (BALQSOs), which display a similarly wide range of ionization states in their winds.
\end{abstract}

Key words: binaries: symbiotic - stars: winds, outflows - accretion, accretion discs — quasars: absorption lines — stars: individual: MWC 560, AS 304

\section{INTRODUCTION}

Accretion onto compact objects exhibits similar phenomenology across a wide range of mass scales, spanning over nine orders of magnitude from supermassive black holes to white dwarfs. In fact, MWC 560 and $\mathrm{CH}$ Cyg - each believed to constitute a white dwarf (WD) accreting from a red giant (RG), the most common kind of symbiotic star-have been called 'nano-quasars,' because broadening their emission lines by a factor of 10 produces optical spectra that almost identically resemble the archetypal Seyfert 1 galaxy I Zw 1 (Zamanov \& Marziani 2002).

MWC 560 also usually exhibits blue-shifted broad absorption lines $\left(\mathrm{BALs}^{1}\right)$ from a jet (Schmid et al. 2001) or

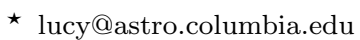

† LSSTC Data Science Fellow

1 We ignore the distinction between BALs and mini-BALs appearing in the quasar context, where the richer sample size sometimes motivates formalism (e.g., Knigge et al. 2008).
}

disk wind. The blue edges of these lines vary between $900-6000 \mathrm{~km} \mathrm{~s}^{-1}$ from the Balmer transitions and combinations of $\mathrm{Al}$ III, $\mathrm{Mg}$ II, Fe II, CriI, Si II, C II, CaII, MgI, $\mathrm{NaI}$, OI, and HeI, including various excited-state, metastable, and resonance transitions (Tomov et al. 1990, 1992; Michalitsianos et al. 1991; Schmid et al. 2001; Meier et al. 1996). MWC 560 thus resembles the rare subset of Fe II lowionization BAL quasars (FeLoBALs) with Balmer BALs; symbiotic nebulae typically have electron densities up to $10^{8}-10^{12} \mathrm{~cm}^{-3}$, comparable to the densities necessary to populate Balmer absorption in photo-ionized quasar winds (Hall 2007; Leighly et al. 2011; Williams et al. 2017). However, MWC 560 has seemed to be missing the set of higherionization ultraviolet (UV) resonance BALs - C IV $\lambda 1550 \AA$, Si IV $\lambda 1400 \AA$, and N v $\lambda 1240 \AA$ — exhibited by many cataclysmic variable (CV) winds (Shlosman \& Vitello 1993) and all BAL quasars outflows (Weymann et al. 1991).

Setting the stage for our analysis, MWC 560's UV spectrum is usually obscured by a curtain of absorption by Fe II and similar ions (Michalitsianos et al. 1991). This iron cur- 




Figure 1.

Top panel: IUE spectra show that MWC 560's iron curtain obscured the UV in 1990 March, vanished in 1990 April, and returned by 1991 September. We re-normalized all spectra to the maximum (1990 April 29) median flux density longward of 3000A, and did not deredden. Line identifications for the 1990 April spectrum are marked at their rest wavelengths.

Middle panel: Predicted optical depth $(\tau)$ relative to the strongest value $\left(\tau_{\max }\right)$, for the Fe II transitions from near the ground state (§ 3.2) in $10 \AA$ bins, excluding higher-excitations populated during iron curtain phases (e.g., on the blue wing of Mg II).

Bottom panel: The Balmer FeLoBAL quasar J172341.1+555340.5 (Hall et al. 2002) resembles the iron curtain states of MWC 560, while the LoBAL quasar J140125.6+581650.7 (Gibson et al. 2009) resembles the 1990 April spectrum (§ 4.3). These Sloan Digital Sky Survey spectra were obtained from the Science Archive Server, corrected for redshift and the negligible Galactic extinction on their sightlines (Green et al. 2015), normalized to match pseudo-continua on an arbitrary scale, and smoothed to resolving power $\mathrm{R}=400$. The FeLoBAL was further dereddened in its rest frame for $\mathrm{E}(\mathrm{B}-\mathrm{V})=0.15$ of SMC bar extinction (Gordon et al. 2003). 
Table 1. Selected IUE spectra of MWC 560 from Michalitsianos et al. (1991), Maran et al. (1991), and Bond et al. (1984).

\begin{tabular}{llcc}
\hline Date $(\mathrm{UT})$ & IUE ID & Bandpass $(\AA)$ & Resolution $(\AA)$ \\
\hline 1984 Mar 10.95 & SWP22459 & $1150-1975$ & $4-7$ \\
1984 Mar 10.93 & LWP02920 & $1910-3300$ & $5-8$ \\
1990 Mar 14.98 & LWP17534 & $1910-3300$ & $5-8$ \\
1990 Mar 14.99 & SWP38361 & $1150-1975$ & $4-7$ \\
1990 Apr 29.90 & SWP38698 & $1150-1975$ & $4-7$ \\
1990 Apr 29.92 & LWP17832 & $1910-3300$ & $5-8$ \\
1990 Apr 29.94 & LWP17833 & $1845-2980$ & $0.1-0.3$ \\
1990 Apr 30.02 & SWP38699 & $1150-1975$ & $4-7$ \\
1991 Sep 29.12 & LWP21366 & $1910-3300$ & $5-8$ \\
1991 Sep 29.14 & SWP42580 & $1150-1975$ & $4-7$ \\
\hline
\end{tabular}

tain weakened dramatically by the end of 1990 April (Skopal 2005), allowing us to see the outflow in a rare, unobscured state. When the next UV spectrum was obtained, on 1990 September 26, the Fe II absorption had returned with even more optical depth than before (Maran et al. 1991).

The paper is structured as follows: We describe the archival data ( $(2)$. We show that a total disruption of the iron curtain in 1990 April unveiled high-ionization UV BALs, which were previously misidentified as Si II and Fe II (§3). We discuss the physical consequences of this findingincluding connections to quasars and novae - and the disappearance of the iron curtain, which has been optically thick at every other epoch $(\S 4.1-\S 4.3)$. The high-ionization BALs link MWC 560 with symbiotic star AS 304, and show that these objects are prototypes for an emerging class of BAL symbiotics ( $§ 4.4)$. Last, we summarize our conclusions $(\S 5)$.

All velocities and wavelengths for MWC 560 are heliocentric.

\section{DATA}

We retrieved NEWSIPS pipeline reductions of several International Ultraviolet Explorer (IUE) large-aperture spectra in

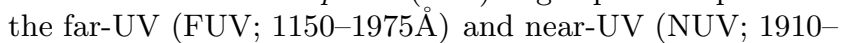
$3300 \AA$ ) , including all UV spectra observed in 1990 April, from the Mikulski Archive for Space Telescopes (MAST). Table 1 lists the spectra, which were previously published in Michalitsianos et al. (1991), Maran et al. (1991), and Bond et al. (1984).

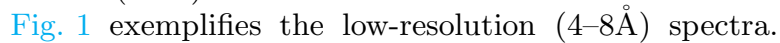
The chosen dates follow Figure 1 in Skopal (2005), which was designed to exhibit the varying depths of MWC 560's iron curtain.

\section{RESULTS: LINE IDENTIFICATION}

\subsection{High-ionization BALs}

The deepest absorption features in the 1990 April 29.90 and 30.02 spectra are located at the expected positions of blue-shifted BALs from the highionization C IV $\lambda 1548.20,1550.77 \AA$, Si IV $\lambda 1393.76,1402.77 \AA$, and N V $\lambda 1238.82,1242.80 \AA$ doublets and He II $\lambda 1640 \AA$ multiplet, as shown by rest-wavelength line-labels in Fig. 1. The

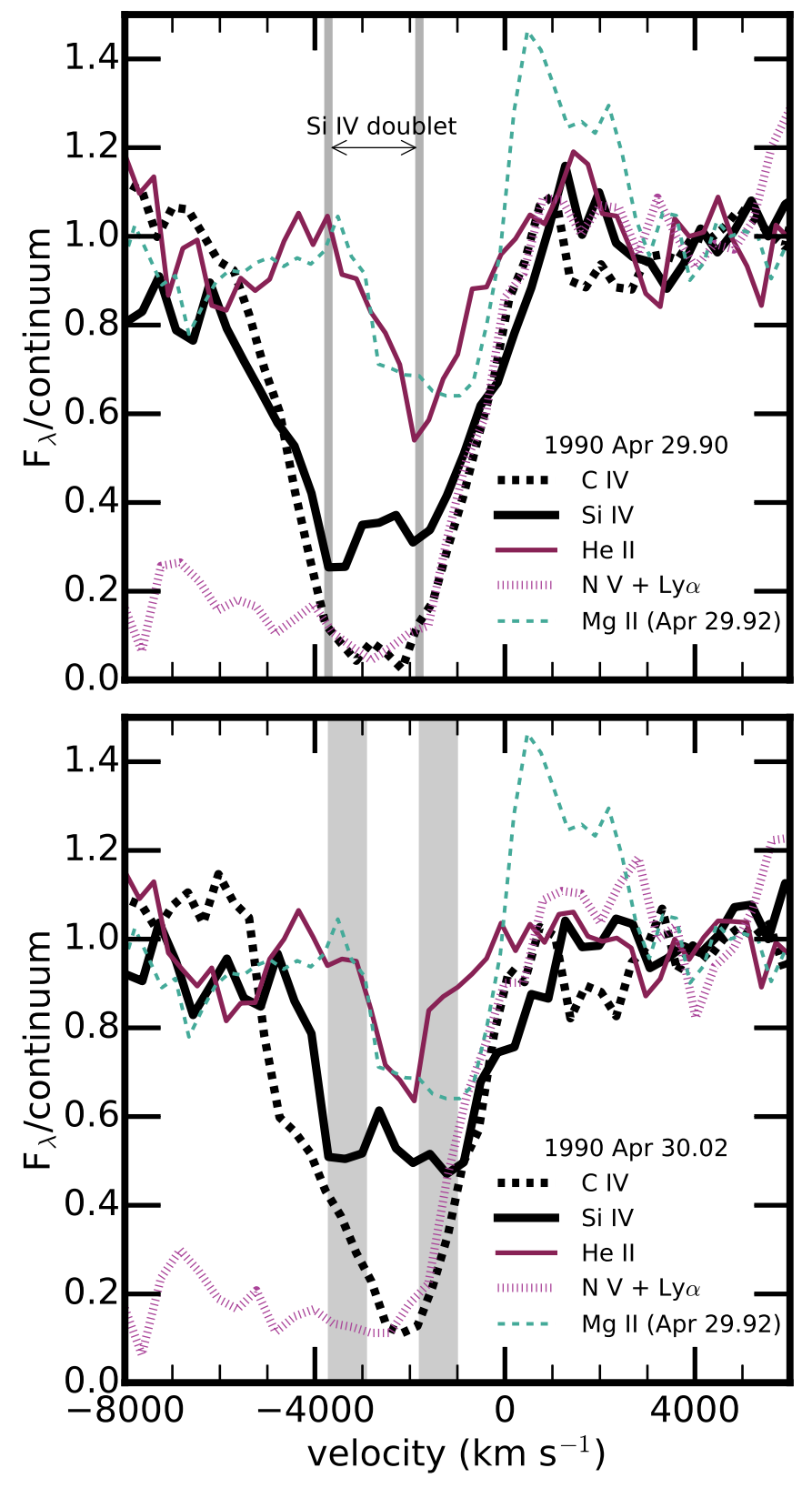

Figure 2. Velocity profiles from the high-ionization BALs in IUE spectra from 1990 April 29.90 (top) and 30.02 (bottom), and Mg II for comparison, with zero-velocity set to the rest wavelength of the reddest transition in each doublet or multiplet (§3.1). The Si IV doublet components are marked at $-1800 \mathrm{~km} \mathrm{~s}^{-1}$ for April 29.90 and -1200 through $-1800 \mathrm{~km} \mathrm{~s}^{-1}$ for April 30.02. Flux density is normalized to the median value between +3000 and $+6000 \mathrm{~km} \mathrm{~s}^{-1}$.

velocity profiles of these strong and rapidly variable BALs are shown in Fig. 2. The Si IV BAL is confirmed by the $9.0 \AA$ (1900 $\left.\mathrm{km} \mathrm{s}^{-1}\right)$ Si IV doublet separation, which is about twice the local full-width-half-max spectral resolution and is observed in the trough (Fig. 2). Subtracting the doublet separations, the CIV and Si IV troughs extend to at least $4000 \mathrm{~km} \mathrm{~s}^{-1}$. N v is contaminated by Ly $\alpha$ absorption at high velocities. He II extends to about $3000 \mathrm{~km} \mathrm{~s}^{-1}$. 



Figure 3. The vertical lines drawn on this IUE spectrum from 1990 April 30.02 are Si II (dashed) and P II (hashed) transition rest wavelengths from Table 2. Atomic physics predicts that the two absorption complexes should be comparably strong, so the comparison region (left) shows that $\mathrm{Si}$ II+P II cannot account for the absorption trough near $1550 \AA$ (right).

Table 2. NIST lab data showing that the $1304 \AA$ and $1526 \AA$ complexes of Si II and P II share similar line strengths: wavelength $\left(\lambda_{l a b}\right)$, lower-level excitation above the ground state $\left(\mathrm{E}_{l}\right)$, oscillator strength $(f)$, and lower-level statistical weight $\left(\mathrm{g}_{l}\right)$.

\begin{tabular}{|c|c|c|c|c|}
\hline Ion & $\lambda_{l a b}(\AA)$ & $\mathrm{E}_{l}(\mathrm{eV})$ & $f$ & $\mathrm{~g}_{l}$ \\
\hline Si II & 1526.71 & 0 & 0.133 & 2 \\
\hline Si II & 1304.43 & 0 & 0.093 & 2 \\
\hline Si II & 1533.43 & 0.036 & 0.133 & 4 \\
\hline Si II & 1309.27 & 0.036 & 0.080 & 4 \\
\hline P II & 1532.53 & 0 & 0.008 & 1 \\
\hline P II & 1301.87 & 0 & 0.038 & 1 \\
\hline $\mathrm{P}_{\text {II }}$ & 1535.92 & 0.020 & 0.006 & 3 \\
\hline $\mathrm{P}_{\mathrm{II}}$ & 1536.42 & 0.020 & 0.002 & 3 \\
\hline $\mathrm{P}_{\text {II }}$ & 1304.49 & 0.020 & 0.013 & 3 \\
\hline $\mathrm{P}_{\text {II }}$ & 1304.68 & 0.020 & 0.009 & 3 \\
\hline P II & 1305.50 & 0.020 & 0.016 & 3 \\
\hline P II & 1542.30 & 0.058 & 0.006 & 5 \\
\hline P II & 1543.13 & 0.058 & 0.001 & 5 \\
\hline $\mathrm{P}_{\text {II }}$ & 1543.63 & 0.058 & 0.000 & 5 \\
\hline P II & 1309.87 & 0.058 & 0.010 & 5 \\
\hline P II & 1310.70 & 0.058 & 0.028 & 5 \\
\hline
\end{tabular}

\subsection{Evidence against prior identifications}

The feature we assign to C IV $\lambda 1550 \AA$ was previously misidentified as Si II near zero-velocity (Michalitsianos et al. 1991; Maran et al. 1991) blended with P II (Maran et al. 1991). Table 2 shows that there is a complex of Si II and P II transitions near $1304 \AA$ with lower levels and oscillator strengths almost identical to those of Si II and P II near $1550 \AA$ A. Fig. 3 shows that the $1304 \AA$ complex is only observed weakly and at large blue-shifts (if at all) in 1990 April. Therefore, Si II and P II cannot produce the deep trough near $1550 \AA$. Moreover, there are no $\mathrm{Si}$ II transitions from close to the ground state near the feature we assign to He II $\lambda 1640 \AA$, further contradicting line labels in Michalitsianos et al. (1991). Our transition data are from the National Institute of Standards and Technology (NIST; Kramida et al. 2017); we checked for additional lines in Kurucz \& Bell (1995).

The lines we assign to $\operatorname{Si}$ IV $\lambda 1400 \AA$ and Nv $\lambda 1240 \AA$ were misidentified as Fe II (Michalitsianos et al. 1991; Maran et al. 1991). In modeling the 1990 March 14 iron curtain, Shore et al. (1994) later speculated that Si IV was probably present. In fact, the situation is clearer in the 1990 April spectra, in which most Fe II absorption has vanished. Any remaining weak lines would be from easily-populated (see Lucy et al. 2014) excitations less than $0.4 \mathrm{eV}$ above the ground state, there being no excitation states between $0.39 \mathrm{eV}$ and $0.98 \mathrm{eV}$ in the $\mathrm{Fe}^{+}$atom. We summed the relative optical depths of these low-excitation lines (oscillator strength $\times$ statistical weight $\times$ wavelength) into $10 \AA$ bins to show that they cannot produce deep FUV absorption without producing deep NUV absorption, which is not observed in 1990 April (Fig. 1). The only sign of iron-like absorption comes from a few of the strongest lowexcitation lines (e.g., Fe II $\lambda 2600 \AA$ ) observed in the highresolution NUV spectrum from 1990 April 29 as zerovelocity, saturated absorption $<1 \AA$ wide; these narrow, possibly-interstellar lines do not produce deep features in the low-resolution spectra. Thus, although low-ionization lines may weakly contaminate the high-ionization BALs on 1990 April 29-30, they cannot account for them.

\section{DISCUSSION}

\subsection{Ionization and velocity structure}

Our results show that photo-ionization is likely a prominent mechanism in the outflow from MWC 560's accretion disk. The broad and continuous velocity distributions of BALs from a wide range of ionization states argue against collisional ionization in discrete shocks or a star-like photosphere alone. Ionizing photons of at least 33,48 , and $77 \mathrm{eV}$ can populate the Si IV, CIV, and Nv BAL transitions, respectively; these are emitted in sufficient quantities at the $\sim 10^{5} \mathrm{~K}$ inner radii of a WD accretion disk with MWC 560's parameters (Schmid et al. 2001).

The presence of high-ionization lines is consistent with the high-velocity absorption from metastable He I* $\lambda 10830 \AA$ in 1990 April 12 and 1991 January 25 infrared spectra of MWC 560 (Meier et al. 1996). This He I* transition's lower level is populated by recombination onto $\mathrm{He}^{+}$; the ionization potentials of neutral helium and $\mathrm{He}^{+}$are 25 and $54 \mathrm{eV}$, re- 
spectively, so metastable He can be partially co-spatial with $\mathrm{C}^{+3}$ and $\mathrm{Si}^{+3}$ (Leighly et al. 2011).

The high-ionization CIV and Si IV BALs exhibit maximum radial velocities at least $1000 \mathrm{~km} \mathrm{~s}^{-1}$ faster than the contemporaneous low-ionization $\mathrm{Mg}$ II $\lambda 2800 \AA$ resonance line (Fig. 2). This behavior is also observed in BAL quasar outflows, for which various explanations have been proposed. Dense, self-shielding clumps may be embedded in a faster, higher-ionization gas (Voit et al. 1993). Equatorial winds can comprise vertically-stratified layers of ionization state and velocity (Matthews et al. 2016). Or an outflow may decelerate along the line of sight, with fast gas close to the photo-ionizing source shielding radially-slower gas closer to the observer (Voit et al. 1993). One or more of these geometries, which are not mutually-exclusive, could apply to MWC 560

The HeII BAL likewise appears to be slower than the C IV and Si IV BALs, probably for a different reason. This behavior is observed in CV and stellar winds, in which the He II $\lambda 1640 \AA$ multiplet's highly-excited lower level (41 eV above the ground state) is thought to be collisionally populated in the hot, dense base of the outflow, where the gas is still accelerating (Hoare 1994; Smith 2006).

\subsection{Vanishing curtains and novae}

Both ground-state and high-excitation $\mathrm{Fe}^{+}$vanished in 1990 April. The iron curtain has been present in every other UV spectrum of MWC 560, including observations in 1984 (Bond et al. 1984), 1990 January-March and late 1990-1993 (Michalitsianos et al. 1991; Skopal 2005), 1995 (IUE PI: Starrfield), and throughout a 2016 high state that rivaled 1990 in bolometric luminosity (Lucy et al., in preparation), suggesting that the disappearance of $\mathrm{Fe}^{+}$signified more than a simple increase in ionizing luminosity.

Instead, the vanishing curtain probably corresponded to a temporary decrease in hydrogen column density-i.e., in total mass along the line of sight. In strongly photo-ionized plasma, iron is distributed between $\mathrm{Fe}^{+2}, \mathrm{Fe}^{+3}$, and $\mathrm{Fe}^{+4}$, making it difficult to form much $\mathrm{Fe}^{+}$even through recombination (see $\S 4.3$ in Lucy et al. 2014). Other 'iron curtain' elements like silicon and chromium (but not magnesium ${ }^{2}$ ) have similar ionization potentials and behave in the same way. Iron curtain ions may survive only when enough mass is present to self-shield some portion of the outflow against high-energy photons.

The decline in column density was likely related to (1) the 1990 January-April optical brightening, which marked both a periodic peak in the system's light curve and a unique, non-periodic, and permanent tripling of the optical luminosity (Leibowitz \& Formiggini 2015; Munari et al. 2016); (2) the dramatic mass ejections of 1990 January-April, which yielded detached BALs blue-shifted up to a system record of $6000 \mathrm{~km} \mathrm{~s}^{-1}$ (Tomov et al. 1990, 1992); and (3) the post-outburst, year-long suppresion of

2 Although $\mathrm{Mg}^{+}$and $\mathrm{Fe}^{+}$have similarly small ionization potentials, $\mathrm{Mg}^{+2}$ can dominate thanks to its large $80 \mathrm{eV}$ ionization potential; recombination can then yield sufficient $\mathrm{Mg}^{+}$for opacity at $2800 \AA$ (Lucy et al. 2014), explaining the persistence of that BAL in MWC 560. optical flickering and slowing of absorption speeds to less than $1000 \mathrm{~km} \mathrm{~s}^{-1}$ in late 1990-1991 (Zamanov et al. 2011b). Coverage of the spectral response to these events is incomplete; to our knowledge, no optical spectra were obtained 1990 April 4 through October 22, and no UV spectra were obtained 1990 May 1 through September 25.

High-ionization UV BALs are briefly observed as P Cygni profiles immediately after an iron curtain phase in some thermonuclear novae (Shore et al. 1993; M. J. Darnley 2018, private communication). The ejected mass rarefies as it expands, erasing the outer, heretofore-shielded, $\mathrm{Fe}^{+}$component. The similarity suggests that MWC 560's wind temporarily turned off in 1990 April after a series of powerful gusts, resulting in the nova-like phenomenology of a discrete mass ejection. This pattern supports the 'discrete' and 'quasistationary' taxonomy proposed by Kolev \& Tomov (1993) for MWC 560's optical absorption lines.

\subsection{A BAL nano-quasar}

To our knowledge, there is no other accretion disk in the Galaxy observed to contain a similar range of ionization and excitation states in a persistent BAL outflow. There are CVs that exhibit both Balmer and CIV BALs (Kafka \& Honeycutt 2004), but we have found no reports of any CV or X-ray binary known to have also exhibited MgII and Fe II BALs except during thermonuclear novae. Unlike novae, Balmer BALs and the iron curtain are almostpermanent properties of MWC 560's spectrum for years at a time. Likewise, the high-ionization UV BALs may be present in every MAST-archived FUV spectrum of MWC 560, including the one obtained by Bond et al. (1984), although contamination by the optically thick iron curtain precludes certainty at all epochs besides 1990 April.

Rather, MWC 560 best resembles Balmer FeLoBAL quasars, wherein photo-ionization models favor high densities in the BAL wind-comparable to densities in symbiotic nebulae $(\S 1)$. These quasar winds persistently exhibit a similarly wide range of ionization states ${ }^{3}$ and similar spectra; see the quasar spectra in Fig. 1. Moreover, iron curtain absorption has recently been observed to vary dramatically and even vanish in some FeLoBAL quasars, albeit on slower timescales than in MWC 560 (Rafiee et al. 2016; see also Hall et al. 2011; McGraw et al. 2015; Zhang et al. 2015; Stern et al. 2017).

\subsection{The BAL symbiotics}

The CIV, Si IV, and Nv BALs in MWC 560 illuminate a link to another symbiotic WD+RG binary: Munari \& Buson (1993) showed that AS 304 contains absorption blue-shifted up to $2200 \pm 400 \mathrm{~km} \mathrm{~s}^{-1}$ from only C IV, Si IV, and Nv. Its FUV spectrum was observed only in 1992, but this observation was not prompted by any particular event, so there is no reason to think that the BALs were transient. The He II emission strength indicated a high luminosity on the order of

3 Except for HeII, whose absence in quasar outflows has been interpreted as a lower limit on the distance of absorbing material from the disk (Wampler et al. 1995). 
$10^{4} \mathrm{~L}_{\odot}$ (Munari \& Buson 1993), which likely required nuclear burning on the surface of the white dwarf to power. The ionization parameter in AS 304's outflow was therefore large, contributing to the absence of low-ionization (and in this case, optical) absorption.

In MWC 560, optical and UV flickering (Bond et al. 1984; Tomov et al. 1996; Zamanov et al. 2011a; Lucy et al., in preparation; though see Zamanov et al. 2011b) and hard X-rays sometimes observed from the boundary layer (Stute \& Sahai 2009) indicate an accretion disk without WD surface burning (see Luna et al. 2013). Thus, both hot burning (AS 304) and cooler non-burning (MWC 560) symbiotics can sustain persistent BAL outflows.

MWC 560 and AS 304 have cousins in symbiotics with transient or low-velocity absorption. $\mathrm{CH}$ Cyg, a non-burning WD+RG binary with optical flickering, exhibited Balmer BALs with blue edges around $2000 \mathrm{~km} \mathrm{~s}^{-1}$ in 2017 January (Iijima 2017); this absorption recurred sporadically throughout the year (Teyssier et al. 2017), sometimes appearing and vanishing within 1.5 hours (T. Iijima 2017, private communication). Z And, a burning-powered WD+RG binary, briefly exhibited a Balmer BAL blue-shifted up to $\approx$ $1700 \mathrm{~km} \mathrm{~s}^{-1}$ on 2006 July 9 (Figure 7 in Tomov et al. 2013), near the peak of a 2 magnitude optical outburst. Meanwhile, many more symbiotics (Tomov et al. 2013) and purported Be stars with cool giant companions (including the iron stars; Cool et al. 2005) exhibit substantially narrower 100$1000 \mathrm{~km} \mathrm{~s}^{-1}$ absorption lines; we will not here attempt to disentangle their heterogeneous population, but some may mimic BAL outflow behaviors (Tomov et al. 2013).

The transient BAL outflows from symbiotics coexist with narrow jets, but the lines of sight to $\mathrm{Z}$ And and $\mathrm{CH}$ Cyg are severely misaligned from any plausible jet axis. Eclipses in $\mathrm{CH}$ Cyg indicate a large inclination angle (Skopal et al. 1996) and the jet is extended in the plane of the sky (Weston 2016, and references therein), suggesting that the jet cannot produce the BALs. We view Z And's orbit at an inclination angle of $47 \pm 12^{\circ}$, and marginally resolved jets have been seen perpendicular to the orbital plane (Brocksopp et al. 2004) and $20^{\circ}$ west of the perpendicular's projection in the sky (Sokoloski et al. 2006; Kenny 1995). Burmeister \& Leedjärv (2007) inferred a $1700 \mathrm{~km} \mathrm{~s}^{-1}$ jet during the 2006 outburst from narrow emission lines at $\pm 1150 \mathrm{~km} \mathrm{~s}^{-1}$, assuming that the jet was perpendicular to the orbit. Speculatively, the similarity between the jet and BAL velocity during this outburst might suggest that the opening angle of a jet-feeding wind briefly spread into the line of sight.

Likewise, the degree to which MWC 560's outflow is collimated could vary with its strength. It probably is a generally polar outflow viewed at a small inclination angle ${ }^{4}$, but BAL quasars and $\mathrm{CV}$ winds show that it is problematic to constrain the degree to which a BAL outflow is collimated just from the ratio of absorption to emission equivalent widths (Turnshek 1997; Hamann et al. 1993; Voit et al. 1993; Drew 1987). BAL-producing outflows with wider opening angles than jets would help to explain the high incidence rate of BAL symbiotics, which now appear to be surprisingly well represented among the $\sim 10$ (e.g., Brocksopp et al. 2004) symbiotics with known jets. Even with large half-opening angles up to $\pm 15^{\circ}$ (as for the R Aqr jet; Schmid et al. 2017), less than $3.5 \%$ of jets should point towards Earth - although a larger fraction might exhibit slower and shallower absorption projected into the line of sight.

Voit et al. (1993) proposed that low-ionization BAL quasars (cf. MWC 560, Z And in outburst, and $\mathrm{CH}$ Cyg) may represent a young evolutionary phase when accretion disk outflows entrain an ambient shroud of dust and gas, which disperses to lower column densities once exclusively high-ionization BALs are observed (cf. AS 304). The dense, frequently $\mathrm{Fe}^{+}$-rich nebulae from cool-giant mass loss in symbiotic binaries (Shore \& Aufdenberg 1993) resemble young quasar environments. We predict that higher-cadence FUV/optical spectral observations and larger sample sizes of both burning and non-burning symbiotics will lead to the detection of more BAL systems than narrow jets alone could produce, and open a window into the physics and geometry of quasar outflows on a nano-scale.

\section{CONCLUSIONS}

A. The FUV spectrum of symbiotic star MWC 560 contains high-ionization BALs from Civ, Si IV, Nv, and HeII (§ 3, Fig. 2). They were unveiled most clearly on 1990 April 29-30, the only time throughout decades of UV observations that MWC 560's curtain of low-ionization iron absorption vanished (Fig. 1).

B. The maximum radial velocities of the $\mathrm{Mg}$ II and He II BALs were at least $1000 \mathrm{~km} \mathrm{~s}^{-1}$ slower than contemporaneous CIV and Si IV BALs. These differences have precedents; $\mathrm{Mg}$ II is slower than C IV and Si IV in BAL quasars, and He II is slower than CIV and Si IV in CV and stellar winds (§ 4.1).

C. The usually-persistent wind in MWC 560 temporarily switched to a discrete ejection phase in 1990. This finding is supported by the phenomenology of some novae, in which the iron curtain lifts to unveil high-ionization UV BALs (§ 4.2).

D. Additional BAL symbiotics exist ( $\S 4.4$ ). The highionization BALs in MWC 560 illustrate a similarity to symbiotic star AS 304, which exhibits exclusively C IV, Si IV, and Nv BALs (Munari \& Buson 1993). Z And and $\mathrm{CH}$ Cyg have exhibited transient Balmer BALs (Tomov et al. 2013; Iijima 2017); these transient BAL symbiotics contain jets, but the jets do not point towards Earth. Wide-angle disk winds are sometimes required to explain the BAL symbiotics, and may account for why the number of symbiotics with BALs is no longer negligible relative to the $\sim 10$ symbiotics with jets.

E. MWC 560 and AS 304 most resemble Balmer FeLoBAL quasars and high-ionization BAL quasars, respectively $(\S 4.3, \S 4.4)$. Both symbiotic star and quasar accretion disks are sometimes embedded in regions of dense gas and dust, so the evolution of these systems may involve similar physics.

${ }^{4}$ See discussion in Schmid et al. (2001) of unpublished echellespectrograph limits on $\mathrm{TiO}$ absorption band motion. 


\section{ACKNOWLEDGEMENTS}

We thank Matt Darnley for insights on connections to novae and editorial comments. ABL thanks Ulisse Munari, Karen Leighly, and Takashi Iijima for other edifying conversations. We thank Michael Rupen, Paul Kuin, Nirupam Roy, Gerardo Juan Manuel Luna, Jennifer Weston, and Peter Somogyi for editorial comments. ABL thanks the LSSTC Data Science Fellowship Program; their time as a Fellow has benefited this work. We are supported by NSF DGE16-44869 (ABL), NSF AST-1616646 (JLS), and Chandra DD6-17080X (ABL, JLS). We gratefully acknowledge our use of pysynphot (STScI Development Team 2013), PyAstronomy (https://github.com/sczesla/PyAstronomy), extinction (Barbary 2016), the Mikulski Archive for Space Telescopes (MAST), the International Ultraviolet Explorer (IUE), the Sloan Digital Sky Survey (SDSS), NASA's Astrophysics Data System (ADS), the SIMBAD database operated at CDS, and the National Institute of Standards and Technology (NIST).

\section{References}

Barbary, K. 2016, extinction v0.3.0, doi:10.5281/zenodo.804967 extinction.readthedocs.io

Bond, H. E., Pier, J., Pilachowski, C., Slovak, M., \& Szkody, P. 1984, in BAAS, Vol. 16, Bulletin of the American Astronomical Society, 516. http://adsabs . harvard.edu/abs/1984BAAS . . 16 . .516B

Brocksopp, C., Sokoloski, J. L., Kaiser, C., et al. 2004, MNRAS, 347, 430. http: //adsabs . harvard.edu/abs/2004MNRAS . 347 . .430B

Burmeister, M., \& Leedjärv, L. 2007, A\&A, 461, L5. http://adsabs . harvard.edu/abs/2007A\%26A . . 461L . . 5B

Cool, R. J., Howell, S. B., Peña, M., Adamson, A. J., \& Thompson, R. R. 2005, PASP, 117, 462. http://adsabs.harvard.edu/abs/2005PASP . .117 . .462C

Drew, J. E. 1987, MNRAS, 224, 595. http://adsabs . harvard.edu/abs/1987MNRAS . 224 . .595D

Fitzpatrick, E. L., \& Massa, D. 2007, ApJ, 663, 320. http://adsabs. harvard.edu/abs/2007ApJ . .663 . .320F

Gibson, R. R., Jiang, L., Brandt, W. N., et al. 2009, ApJ, 692, 758 http://adsabs.harvard.edu/abs/2009ApJ . . 692 . .758G

Gordon, K. D., Clayton, G. C., Misselt, K. A., Landolt, A. U., \& Wolff, M. J. 2003, ApJ, 594, 279 http://adsabs.harvard.edu/abs/2003ApJ...594..279G

Green, G. M., Schlafly, E. F., Finkbeiner, D. P., et al. 2015, ApJ, 810, 25 http: //adsabs.harvard.edu/abs/2015ApJ . .810. . 25G

Hall, P. B. 2007, AJ, 133, 1271. http://adsabs .harvard.edu/abs/2007AJ . . .133.1271H

Hall, P. B., Anderson, S. F., Strauss, M. A., et al. 2002, ApJS, 141, 267. http://adsabs . harvard.edu/abs/2002ApJS . .141 . .267H

Hall, P. B., Anosov, K., White, R. L., et al. 2011, MNRAS, 411, 2653 http: //adsabs.harvard.edu/abs/2011MNRAS.411.2653H

Hamann, F., Korista, K. T., \& Morris, S. L. 1993, ApJ, 415, 541. http://adsabs . harvard.edu/abs/1993ApJ . . 415 . .541H

Hoare, M. G. 1994, MNRAS, 267, 153 http://adsabs . harvard.edu/abs/1994MNRAS . 267 . .153H

Iijima, T. 2017, The Astronomer's Telegram, 10142. http://adsabs.harvard.edu/abs/2017ATel10142....1I

Kafka, S., \& Honeycutt, R. K. 2004, AJ, 128, 2420. http://adsabs.harvard.edu/abs/2004AJ....128.2420K
Kenny, H. T. 1995, PhD thesis, University of Calgary (Canada). http://adsabs . harvard. edu/abs/1995PhDT.......25K

Knigge, C., Scaringi, S., Goad, M. R., \& Cottis, C. E. 2008, MNRAS, 386, 1426 http://adsabs . harvard. edu/abs/2008MNRAS. 386.1426K

Kolev, D., \& Tomov, T. 1993, A\&AS, 100, 1. http://adsabs. harvard.edu/abs/1993A\%26AS . .100 . . .1K

Kramida, A., Yu. Ralchenko, Reader, J., \& and NIST ASD Team. 2017, NIST Atomic Spectra Database (ver. 5.5.1), [Online]. Available: https://physics.nist.gov/asd [2017 November]. National Institute of Standards and Technology, Gaithersburg, MD.

Kurucz, R. L., \& Bell, B. 1995, Atomic Line Data, Kurucz CD-ROM No. 23, Cambridge, Mass.: Smithsonian Astrophysical Observatory. https://www. cfa.harvard.edu/amp/ampdata/kurucz23/sekur.html

Leibowitz, E. M., \& Formiggini, L. 2015, AJ, 150, 52. http://adsabs.harvard.edu/abs/2015AJ ...150 ...52L

Leighly, K. M., Dietrich, M., \& Barber, S. 2011, ApJ, 728, 94. http://adsabs.harvard.edu/abs/2011ApJ . . 728 . . 94L

Lucy, A. B., Leighly, K. M., Terndrup, D. M., Dietrich, M., \& Gallagher, S. C. 2014, ApJ, 783, 58. http://adsabs . harvard.edu/abs/2014ApJ . . 783. . .58L

Luna, G. J. M., Sokoloski, J. L., Mukai, K., \& Nelson, T. 2013, A\&A, 559, A6. http://adsabs.harvard.edu/abs/2013A\%26A...559A...6L

Maran, S. P., Michalitsianos, A. G., Oliversen, R. J., \& Sonneborn, G. 1991, Nature, 350, 404. http://adsabs. harvard.edu/abs/1991Natur. 350 . 404M

Matthews, J. H., Knigge, C., Long, K. S., et al. 2016, MNRAS, 458, 293 http://adsabs . harvard . edu/abs/2016MNRAS . 458. .293M

McGraw, S. M., Shields, J. C., Hamann, F. W., et al. 2015, MNRAS, 453, 1379 http://adsabs . harvard. edu/abs/2015MNRAS . 453.1379M

Meier, S. R., Rudy, R. J., Lynch, D. K., et al. 1996, AJ, 111, 476. http://adsabs .harvard.edu/abs/1996AJ....111..476M

Michalitsianos, A. G., Maran, S. P., Oliversen, R. J., et al. 1991, ApJ, 371, 761. http://adsabs . harvard.edu/abs/1991ApJ ...371 . .761M

Munari, U., \& Buson, L. M. 1993, MNRAS, 263, 267. http://adsabs .harvard.edu/abs/1993MNRAS . 263. .267M

Munari, U., Dallaporta, S., Castellani, F., et al. 2016, New Astron., 49, 43. http: //adsabs.harvard.edu/abs/2016NewA...49..43M

Rafiee, A., Pirkola, P., Hall, P. B., et al. 2016, MNRAS, 459, 2472 http://adsabs . harvard. edu/abs/2016MNRAS . 459.2472R

Schmid, H. M., Kaufer, A., Camenzind, M., et al. 2001, A\&A, 377, 206. http://adsabs.harvard.edu/abs/2001A\%26A...377. .206S

Schmid, H. M., Bazzon, A., Milli, J., et al. 2017, A\&A, 602, A53. http://adsabs . harvard.edu/abs/2017A\%26A . . 602A . .53S

Shlosman, I., \& Vitello, P. 1993, ApJ, 409, 372. http://adsabs. harvard.edu/abs/1993ApJ . . 409. . 372S

Shore, S. N., \& Aufdenberg, J. P. 1993, ApJ, 416, 355. http://adsabs .harvard.edu/abs/1993ApJ . . 416 . .355S

Shore, S. N., Aufdenberg, J. P., \& Michalitsianos, A. G. 1994, AJ, 108, 671 http: //adsabs . harvard.edu/abs/1994AJ ....108. .671S

Shore, S. N., Sonneborn, G., Starrfield, S., RiestraGonzalez, R., \& Ake, T. B. 1993, AJ, 106, 2408. http://adsabs . harvard.edu/abs/1993AJ....106.2408S

Skopal, A., Bode, M. F., Lloyd, H. M., \& Tamura, S. 1996, A\&A, 308, L9 http://adsabs . harvard.edu/abs/1996A\%26A . . 308L . . .9S

Skopal, A. 2005, A\&A, 440, 995. http://adsabs. harvard.edu/abs/2005A\%26A . .440. .995S

Smith, M. A. 2006, A\&A, 459, 215. 
http: //adsabs.harvard.edu/abs/2006A\%26A. . 459. .215S

Sokoloski, J. L., Kenyon, S. J., Espey, B. R., et al. 2006, ApJ, 636, 1002. http://adsabs.harvard.edu/abs/2006ApJ . .636.1002S

Stern, D., Graham, M. J., Arav, N., et al. 2017, ApJ, 839, 106 http: //adsabs . harvard.edu/abs/2017ApJ . . 839 . 106S

STScI Development Team 2013, Astrophysics Source Code Library, ascl:1303.023 https://pysynphot.readthedocs.io

Stute, M., \& Sahai, R. 2009, A\&A, 498, 209. http: //adsabs.harvard.edu/abs/2009A\%26A...498..209S

Teyssier, F., Shore, S., Berardi, P., et al. 2017, ARAS Eruptive Stars Information Letter, $\mathrm{n}^{\circ} 36$. http://www.astrosurf.com/aras/novae/InformationLetter/ARAS_EruptiveStars_2017-07.pdf

Tomov, N. A., Tomova, M. T., \& Bisikalo, D. V. 2013, in American Institute of Physics Conference Series, Vol. 1551, American Institute of Physics Conference Series, ed. I. Zhelyazkov \& T. M. Mishonov, 30-45. http://adsabs.harvard.edu/abs/2013AIPC.1551_..30T

Tomov, T., Kolev, D., Zamanov, R., Georgiev, L., \& Antov, A. 1990, Nature, 346, 637. http://adsabs.harvard.edu/abs/1990Natur.346. .637T

Tomov, T., Zamanov, R., Kolev, D., et al. 1992, MNRAS, 258, 23. http://adsabs . harvard.edu/abs/1992MNRAS . 258 . . 23T

Tomov, T., Kolev, D., Ivanov, M., et al. 1996, A\&AS, 116, 1. http: //adsabs.harvard.edu/abs/1996A\%26AS . 116 . . .1T

Turnshek, D. A. 1997, in Astronomical Society of the Pacific Conference Series, Vol. 128, Mass Ejection from Active Galactic Nuclei, ed. N. Arav, I. Shlosman, \& R. J. Weymann, 52. http: //adsabs.harvard.edu/abs/1997ASPC. .128...52T

Voit, G. M., Weymann, R. J., \& Korista, K. T. 1993, ApJ, 413, 95. http://adsabs.harvard.edu/abs/1993ApJ . .413 . .95V

Wampler, E. J., Chugai, N. N., \& Petitjean, P. 1995, ApJ, 443, 586. http: //adsabs.harvard.edu/abs/1995ApJ . . 443 . 586W

Weston, J. H. S. 2016, PhD thesis, Columbia University, doi:10.7916/D87M082M. http://adsabs.harvard.edu/abs/2016PhDT .......30W

Weymann, R. J., Morris, S. L., Foltz, C. B., \& Hewett, P. C. 1991, ApJ, 373, 23. http: //adsabs.harvard.edu/abs/1991ApJ . . 373 . . 23W

Williams, R. J., Maiolino, R., Krongold,

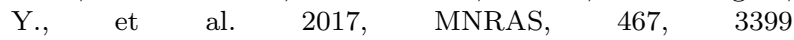
http: //adsabs . harvard.edu/abs/2017MNRAS . 467.3399W

Zamanov, R., Boeva, S., Latev, G., et al. 2011a, Information Bulletin on Variable Stars, 5995. http: //adsabs.harvard.edu/abs/2011IBVS.5995 ...1Z

Zamanov, R., \& Marziani, P. 2002, ApJ, 571, L77. http://adsabs.harvard.edu/abs/2002ApJ . . 571L . .77Z

Zamanov, R. K., Tomov, T., Bode, M. F., et al. 2011b, Bulgarian Astronomical Journal, 16, 3. http: //adsabs.harvard.edu/abs/2011BlgAJ . .16 . . .3Z

Zhang, S., Zhou, H., Wang, T., et al. 2015, ApJ, 803, 58 http://adsabs.harvard.edu/abs/2015ApJ . .803 . .58Z

This paper has been typeset from a $\mathrm{T}_{\mathrm{E}} \mathrm{X} / \mathrm{LAT} \mathrm{E}$. file prepared by the author. 